\title{
INFLUENCE OF SEED PRIMING ON GROWTH AND SEED YIELD OF OKRA UNDER WATER STRESS
}

\author{
HEGAZI, AMAL $Z$.
}

Horticulture Research Institute, ARC, Giza, Egypt

Corresponding author email: hegaziamal@gmail.com

(Manuscript received 12 February 2014)

\begin{abstract}
Seed priming is a technique that improves seed performance in the field and hence ameliorates subsequent germination, growth and seed yield. Moreover, seed priming is often implicated in improving the stress-tolerance of germinating seeds. This study was initiated to determine the seed yield and quality of a local genotype okra var. Sabahia in relation to seed priming and water stress. Two field experiments were conducted during the summer seasons 2012 and 2013 at Kaha (Kalyiobia Governorate), Horticulture Research Station, Egypt. The experimental design used is split plot with four replications. The main plots represented two irrigation regimes either normal or stress 20 days or 30 days intervals respectively, whereas the priming treatments represented the subplots. For priming treatments, seeds were soaked for 24 hours either in water (hydropriming) or in a $3 \%$ solution of $\mathrm{Na}_{2} \mathrm{HPO}_{4}, \mathrm{MgSO}$, or $\mathrm{KCl}$ (osmopriming), whereas dry seeds represented the control.

Results indicated that all plant traits determined were reduced under water stress while this reduction was improved in the primed seeds. Using $\mathrm{Na}_{2} \mathrm{HPO}_{4}$ or $\mathrm{MgSO}_{4}$ seed priming treatments under both water regimes applied gave the highest and best results regarding plant growth, seed yield and quality. Obtained results also showed that seed priming with $\mathrm{KCl}$ gave lower positive effects in this concern and such results seemed to be higher than those obtained by hydropriming treatments. In addition, results clearly showed that okra plants could resist the applied water stresses treatments as a result of seed priming processes used which were found to cause accumulation of some osmolytes as proline.

Data of the present work showed proline accumulation in response to water stress, which might assume that this osmolyte could be enhanced by seed priming. Generally, it could be concluded that seed priming results in improving growth and seed criteria of okra especially under water stress conditions.
\end{abstract}

Key words: Okra, osmopriming, hydropriming, water stress, seed yield \& quality, proline. 


\section{INTRODUCTION}

Uniform and fast germinating seeds are of prime importance for agriculture. To improve the germination properties of seeds, different treatments are used including priming (Badek et al. 2006). Seed priming is a technique that involves the controlled hydration of seeds and has beneficial effects such as enhanced velocity and uniformity of seedling emergence and increased seed tolerance to unfavorable environmental conditions (Lima and Marcos 2010). It involves imbibition of seeds in water under controlled conditions up to the point of radical emergence followed by drying the seed back to its initial moisture content (Ratikanta 2011).

Out of several methods of seed priming, two of them namely hydropriming (soaking seeds in water) and osmopriming (soaking seeds in any osmotic solution of inorganic salts such as $\mathrm{KNO}_{3}, \mathrm{~K}_{3} \mathrm{PO}_{4}, \mathrm{KH}_{2} \mathrm{PO}_{4}, \mathrm{NaNO}_{3}, \mathrm{MnSO}_{4}, \mathrm{MgSO}_{4}, \mathrm{Na}_{2} \mathrm{HPO}$, $\mathrm{KCl}$ and $\mathrm{MnCl}_{2}$ are more prominent. This technique had been applied by many scientists on okra planting because okra seed have hard seed coat (and hence not promptly germinating after planting) which cause major problem to okra growers. Okra is an important crop in temperate and tropical climates. The seeds can be a source of antioxidant, which is essential in maintaining health. Okra flour has huge potential for use to enrich foods in order to provide adequate nutritional daily needs (Adelakun and Oyelade 2011). Okra pods can be consumed in many ways as fresh (raw), dried, cooked, frozen, fried and pickled. Average mineral concentrations in raw and cooked okra (in $\mathrm{mg} / 100 \mathrm{~g}$ ) range from 366 to $325(\mathrm{Ca}) ; 0.102$ to $0.052(\mathrm{Cu}) ; 267$ to $97.7(\mathrm{~K})$; 45.3 to $18.3(\mathrm{Mg}) ; 18.3$ to $7.00(\mathrm{Na}) ; 44.5$ to $25.8(\mathrm{P})$; and 0.233 to $0.094(\mathrm{Zn})$ (Ivanice et al. 2013).

In a trail conducted at Pakistan, soaking okra seeds in a single super phosphate (SSP) solution induced a higher germination percentage (85.94\%), survival percentage $(94.05 \%)$, plant height $(138.97 \mathrm{~cm})$, number of leaves $(26.69)$, number of pods (31.01), seeds/ pod (49.52), pod length $(10.99 \mathrm{~cm})$, pod yield $(2702.69 \mathrm{~kg} / \mathrm{ha})$, early emergence (7.79) and flowering (33.65). The highest plant weight (433.36 g) was observed in plots where seeds soaked with diammonium phosphate (DAP) were used (Shah et al. 2011). Another experiment carried out in Swaziland by Sikhondze and Ossom (2011) aimed to determine how long okra seeds should be primed in order to influence seedling growth and development. Four time durations $(6,12,24$, or 36 h) were used for hydro priming okra seeds. Seedlings grown from seeds primed for 24 hours had the greatest mean length and mean stem diameter, as compared with those of the control (non primed seeds). The authors recommended priming okra seeds for 24 hours before planting. Hydropriming capsicum seeds for 0 to $12 \mathrm{~h}$ also 
resulted in lower days to emergence. An increase in hydropriming durations resulted in an increase in the seedling emergence, energy of emergence, seedling vigor and emergence index. Therefore, the study suggested the use of hydropriming as a simple and cost-effective strategy in pepper production, especially in developing countries (Adebisi et al. 2013).

Moreover, seed priming is often implicated in improving the stress-tolerance of germinating seeds. A hypothetical model illustrating the cellular physiology of priminginduced stress-tolerance suggested achievement of two strategies (Chen and Arora 2013). First, seed priming sets in motion germination-related activities that facilitate the transition of quiescent dry seeds into germinating state and lead to improved germination potential. Secondly, priming imposes a biotic stress on seeds that repress radicle protrusion but stimulates stress responses, potentially inducing crosstolerance. Together, these two strategies constitute a 'priming memory' in seeds, which can be recruited upon a subsequent stress-exposure and mediates greater stress-tolerance of germinating primed seeds. Osmopriming cumin seeds $(-0.8$ and -1.2 Mpa of $\mathrm{PEG}_{6000}$ solution) accelerated seed germination to a largest extent and improved the germination rate and uniformity under drought stress, especially at $15^{\circ} \mathrm{C}$ incubation as compared to $10^{\circ} \mathrm{C}$ and $25^{\circ} \mathrm{C}$. Improved stress tolerant was evident as enhanced germination performance at 10,15 and $25^{\circ} \mathrm{C}$ and under water stress of -0.4 and $-0.8 \mathrm{Mpa}$ of $\mathrm{PEG}_{6000}$ solution (Rahimi 2013).

Thus, the present work intended to study the effect of seed hydro- and osmo- priming of a local genotype of okra (Sabahia) on the plant growth, seed yield and seed quality under water stress conditions.

\section{MATERIALS AND METHODS}

Pure lot of okra seeds (Abelmoschus esculentus L.) Moench, variety Sabahia (green pods) was obtained from the Vegetable Crops Seed Production and Technology Department, Horticulture Research Institute, Agriculture Research Center, Egypt. Two field experiments were conducted during two successive summer seasons of 2012 and 2013 at Kaha (Kalyiobia governorate) Horticulture Research Station, Egypt. Sowing was done on the $1^{\text {st }}$ week of April in the two successive seasons of the present work.

The experiments were laid out in a split plot design with four replicates. The main plots were devoted to two irrigation regimes: normal 20 days or stress 30 days intervals, respectively. The field capacity was $30 \%$ at ordinary irrigation type and reached around $40 \%$ at water stress. The whole area was irrigated after 21 days from sowing then the two irrigation regime were applied. The sub-plots consisted of five 
treatments as follows: 1) dry seeds as control, 2) hydroprimed seeds soaked in water, $3), 4$ ) and 5) representing osmoprimed seeds soaked in $\mathrm{Na}_{2} \mathrm{HPO}_{4}, \mathrm{MgSO}_{4}$, or $\mathrm{KCl} 3 \%$ each, on the bases of a preliminary experiment. Soaking time was 24 hours at $25 \mathrm{CO}^{\circ}$ (room temperature) and the priming solution represented double the volume of seeds. After priming, the seeds were rinsed thoroughly, surface dried at room temperature and kept until planting.

Seeds of each treatment were planted in 6 rows ( $5 \mathrm{~m}$ long and $0.6 \mathrm{~m}$ wide) of 18 $\mathrm{m}^{2}$. Hills were $30 \mathrm{~cm}$ apart with 3 seeds per each. Thinning was done three weeks later to 1 plant/ hill. Other routine agricultural practices such as: irrigation, weeding, fertilization and pest control were carried out as recommended.

\section{Measurements}

\section{Vegetative growth characters}

The following growth attributes were measured after six weeks using ten random plants from each treatment: plant height $(\mathrm{cm})$, number of leaves/plant, leaves area/plant $\left(\mathrm{cm}^{2}\right)$, plant fresh and dry weights $(\mathrm{g})$. For estimating the relative water content (RWC), the fourth leaf from the plant top was taken from three randomly selected plants in each treatment. The RWC was calculated according to the following equation as cited after Barras and Weatherley (1962).

$$
\mathrm{RWC}=\frac{\mathrm{FW}-\mathrm{DW}}{\mathrm{TW}-\mathrm{DW}} \times 100
$$

Where: $F W=$ in situ fresh weight of leaf discs, $D W=$ dry weight of discs at $75 C^{\circ}$ for $48 \mathrm{~h}$.

Full-turgor weight (TW) was determined by floating leaf discs (1cm diameter) on distilled water for $6 \mathrm{~h}$ in Petri dishes under lab conditions and then blotted before weighting.

\section{Seed yield, seed quality and its components}

At harvest, samples of ten random plants from each treatment were used to record the following characters: number of branches/plant, number of pods/plant, number of seeds/ pod, seeds weight/plant (g), weight of 100 seeds (seed index) and total seed yield/plot (kg).

\section{Chemical analyses}

The top fourth leaf from 10 random plants were picked up (after five weeks) and washed by distilled water for the quantitative colorimetric determination of chlorophylls and carotenoids. Total nitrogen was determined in seeds using microKjeldahl apparatus. Phosphorus was also colorimetrically estimated. Potassium was determined using flame-photometer. Those analyses were assayed according to 
A.O.A.C (2000). Extraction and calorimetric determination of proline were done according to Bates et al. (1973).

\section{Germination potential of the yielded seeds}

Three random samples (100 seeds each) were used from each treatment for calculating the following records;: germination percentage (\%), germination rate, seedling and root length $(\mathrm{cm})$. Germination rate was calculated according to as following equation:

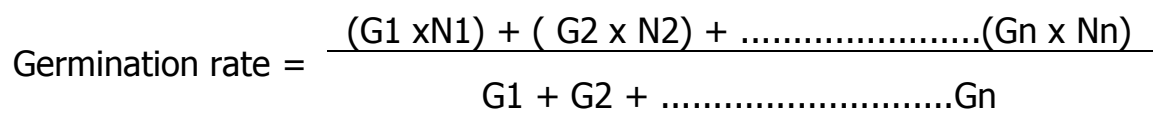

Where: $\mathrm{G}=$ Number of germinated seeds in certain day, $\mathrm{N}=$ Number of this certain day

\section{Statistical Analysis:}

All data were tabulated and statistically analyzed using the analyses of variance method as reported by Snedecor and Cochran. Duncan's multiple range at $5 \%$ level of probability was calculated to compare between means, as shown by Dospekhov (1984).

\section{RESULTS AND DISCUSSION}

\section{Vegetative growth}

The data presented in Table 1-a reveal that all vegetative characters of okra significantly decreased under water stress except plant height on the $1^{\text {st }}$ season which was not significantly affected by the applied water stresses. All seed priming solutions induced significant increases in the growth criteria of the produced plants, with best results on osmopriming using $\mathrm{Na}_{2} \mathrm{HPO}_{4}$. Interaction between irrigation type and seed priming (Table1-b) indicated that all priming solutions $\left(\mathrm{Na}_{2} \mathrm{HPO}_{4}, \mathrm{MgSO}_{4}\right.$ and $\left.\mathrm{KCl}\right)$ showed significant increases over the control (dry seeds) or the plants resulting from hydropriming in both seasons with the two applied irrigation regimes. Under normal irrigation regime, $\mathrm{Na}_{2} \mathrm{HPO}_{4}$ showed high values for all criteria illustrated in table 1-b but under water stress, $\mathrm{MgSO}_{4}$ gave higher values for plant height and fresh\& dry weight/ plant. Response of okra seeds to seed priming and its effects on growth parameters of okra plants were studied by Shah et al. (2011). They stated that okra seeds primed for $24 \mathrm{~h}$ either in diammonium phosphate (DAP) or single super phosphate (SSP) gave higher plant height, number of leaves and plant weight. The SSP solution performed best with most parameters, while distilled water showed poor performance. These conclusions are in agreement with the obtained results indicated in Table 1-a\&b which showed that osmopriming of okra seeds was more efficient than hydropriming in improving vegetative growth. 
To understand the physiology of seed osmopriming and subsequent stress tolerance of the resulting plants, Chen and Arora (2011) primed spinach seeds with $-0.6 \mathrm{MPa}$ PEG at $15^{\circ} \mathrm{C}$ for $8 \mathrm{~d}$ then unprimed and primed germinating seeds/ seedlings were subjected to chilling and desiccation stresses. They indicated that during osmopriming, the transition of seeds from dry to germinating state represses the antioxidant pathways (residing in dry seeds) but stimulates another pathway (only detectable in imbibed seeds). They added that osmopriming strengthens the antioxidant system and increases seed germination potential, resulting in an increased stress tolerance in germinating seeds.

\section{Photosynthetic pigments}

Data presented in Table 2-a indicate that under water stress, a significant decrease in all photosynthetic pigment contents except chlorophyll $b$, which showed $a$ significant increase in both seasons. Data also showed that, no clear trend was noticed between seed treatments in both seasons of the experiment, regarding the photosynthetic pigment contents of the plant leaves (Table 2-a). Concerning the interaction between seed priming treatments and water regime, Table 2-b indicated that all treatments showed a generally significant increase comparing with control (dry seed) under both irrigation types. This trend clearly appeared in total pigments contents, where lowest values $\left(13.41,14.10 \mathrm{mg} / \mathrm{g} \mathrm{f}\right.$. wt., for the $1^{\text {st }}$ and second seasons, respectively) were obtained in case of unprimed seeds. The priming enhancement effect of photosynthetic pigments has been suggested to be attributed to an improved germination performance and plant tolerance under temperature or water stress that would be reflected as enhanced growth (Chen et al. 2010). This could be further reinforced where stomatal conductance and relative chlorophyll contents of melon plants resulting from primed seeds were almost higher than those of the corresponding unprimed ones (Sivritepe et al. 2005).

\section{Seed yield}

Results obtained in Table 3-a demonstrate significantly decreased non consistent differences in the seed yield traits under the influence of the two water stresses used. Effects of hydro- and osmo- priming on seed yield indicated that $\mathrm{Na}_{2} \mathrm{HPO}_{4}$ gave best significant effects, as compared with the control (Table 3-a). In this respect, $\mathrm{MgSO}_{4}$ gave the second higher values followed by $\mathrm{KCl}$. On the other hand, hydropriming resulted in the lowest values, compared with other seed treatments; however being higher than the control. Interaction between types of irrigation and seed treatments (Table3-b) indicated that the three osmopriming solutions under investigation gave the best significant results for all seed yield characters under normal irrigation. In addition, the yield traits shown on using $\mathrm{Na}_{2} \mathrm{HPO}_{4}$ under stress were approximately 
comparable to those obtained with other priming solutions under normal condition, except with the weight of 100 seeds (Table 3-b). There were no significant differences between the effects of seed priming with $\mathrm{MgSO}_{4}$ and $\mathrm{KCl}$, with regard to the number of pods/ plant, pod length $(\mathrm{cm})$ and wt. of 100 seeds $(\mathrm{g})$ in case of normal irrigation, whereas non significant differences between the above mentioned two osmo-priming treatments in the number of seeds/ pod and weight of 100 seed (g) under stress.

Enhancement of plant vigor and subsequently its yield by seed priming was detected by many authors. Thus, when okra seeds were primed in: distilled water or diammonium phosphate (DAP), single super phosphate (SSP), or $\mathrm{SSP}+\mathrm{Na}_{2} \mathrm{CO}_{3}$ for $4 \mathrm{~h}$ and its folds up to $48 \mathrm{~h}$, there was significantly increase in plant height, number of leaves, and pods per plant, pod length, number of seeds per pod and pod yield (Shah et al. 2011)[7]. In addition, priming of red bean seeds in $100 \mathrm{ppm} \mathrm{KCl}, 0.5 \% \mathrm{CaCl}_{2}$, or

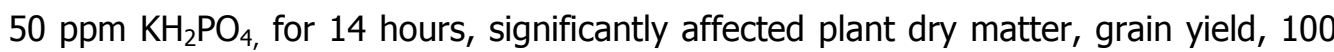
grain weight and number of pods (Rastin et al. 2013).

To understand priming benefits and its association with post-priming stress tolerance, Chen and Arora (2011) had been concluded that osmopriming strengthens the antioxidant system and increases seed germination potential, resulting in an increased stress tolerance in germinating seeds. In this concern, Chen et al. (2010) found that priming improved spinach seed stress tolerance by improving germination performance at water stress of -0.8 and $-1.2 \mathrm{MPa}$.

\section{Chemical content of seeds}

From the data illustrated in Table 4-a a significant decrease in N,P and $\mathrm{K}$ content of seeds is observed as a result of water stress, while the content of proline is significantly increased. All priming treatments induced a significant increase over the control (and in some cases over the records with hydro-priming) for N, P, K and proline contents. Interaction between irrigation types and seed treatments (Table 4-b) showed that the content of NPK increased as a result of seed priming under both normal and water stress treatments and this increase was greater under normal irrigation. But, this trend was reversible in case of proline i.e. its content was markedly increased under water stress.

Increased nutrient contents in primed seeds was observed by Farooq et al. (2010). They stated that seed priming changed the pattern of $\mathrm{N}$ and $\mathrm{Ca}^{2+}$ homeostasis both of the seeds and seedlings, which were associated with enhanced a-amylase activity. They suggested that as a result of seed priming, most of $\mathrm{N}$ and $\mathrm{Ca}^{2+}$ were partitioned to the embryo, which enhanced seedling emergence and subsequent growth of rice seedlings. In addition, an improvement in phosphorus, calcium, and potassium 
contents were observed from osmohardening plant seeds with $\mathrm{CaCl}_{2}$ followed by $\mathrm{KCl}$ (Rehmani et al. 2011). Accumulation of proline as a result of seed priming appears to be a promising approach to maintain the productivity of plants under stress condition. In this connection, it has been found that seed priming led to increased soluble carbohydrate and proline contents and decreased seed membrane damage of muskmelon cultivars because malondialdehyde (MDA) concentration was low in primed group in contrast to the nonprimed one (Farhoudi et al. 2011). Also, total soluble proteins and proline continued to increase under drought in cultivars of hot pepper. So, the plants were better able to resist drought by better growth and yield due to higher accumulation of osmolytes and maintenance of the tissue water contents (Anjum et al. 2012).

\section{Seed quality}

Data presented in Table 5 -a reveal that germination and seedling growth decreased under water stress, with a significantly increased germination rate i.e. the seed took longer time to complete germination. As regard the interaction between irrigation regimes used and seed priming, data of Table 5 -b indicated that seed quality criteria were significantly affected by this interaction. Seed priming with $\mathrm{Na}_{2} \mathrm{HPO}_{4}$ was found to be the best treatment in this concern followed by $\mathrm{MgSO}_{4}$ and then $\mathrm{KCl}$. Enhancement of seedling growth vigor by priming was detected by many authors. Different osmopriming treatments of pulses gave high and reliability significance in germination (\%), growth vigor index and shoot and root criteria under water deficit condition (Rajavel and Vincent 2009). osmopriming of mung bean using $\mathrm{KHPO}_{4}$ at $0.60 \%$ significantly improved seed vigor and final germination percentage (Umair et al. 2010). In addition, seed priming of spinach has been suggested to improve germination performance under temperature or water stress (Chen et al. 2010).

\section{CONCLUSION}

It could be concluded that, pre-sowing treatments either by soaking okra seeds for $24 \mathrm{~h}$ in water (hydropriming) or in $3 \%$ of $\mathrm{Na}_{2} \mathrm{HPO}_{4}$ or $\mathrm{KCl}$ or $\mathrm{MgSO}_{4}$ (osmopriming) could improve plant growth, seed yield and seed quality of pkra especially under water stress conditions. Priming with $\mathrm{Na}_{2} \mathrm{HPO}_{4}$ was the best treatment regarding all the studied criteria followed by $\mathrm{MgSO}_{4}$ then $\mathrm{KCl}$. Hydropriming had lower effectiveness but exhibited better effects than the control. 
Table (1-a). Effect of irrigation type (normal\& water stress) and seed priming for $24 \mathrm{~h}$ either in water (hydropriming) or in $3 \% \mathrm{Na}_{2} \mathrm{HPO}_{4}$, $\mathrm{MgSO}{ }_{4}$, or $\mathrm{KCl}$ solution (osmopriming) on growth criteria of okra (var. Sabahia) plants during summer seasons 2012, 2013. RWC= Relative water contents.

\begin{tabular}{|c|c|c|c|c|c|c|c|c|c|c|c|c|}
\hline \multirow{2}{*}{$\begin{array}{l}\text { Character } \\
\text { Treatments }\end{array}$} & \multicolumn{2}{|c|}{ Plant height $(\mathrm{cm})$} & \multicolumn{2}{|c|}{ No. of leaves/ plant } & \multicolumn{2}{|c|}{$\begin{array}{c}\text { Mean area of leaves / } \\
\text { plant }\left(\mathrm{cm}^{2}\right)\end{array}$} & \multicolumn{2}{|c|}{ Fresh weight/ plant ( $\mathrm{g}$ ) } & \multicolumn{2}{|c|}{ Dry weight/ plant $(\mathrm{g})$} & \multicolumn{2}{|c|}{ RWC } \\
\hline & $\begin{array}{c}1^{\mathrm{st}} \\
\text { Season } \\
\end{array}$ & $\begin{array}{c}2^{\text {nd }} \\
\text { Season } \\
\end{array}$ & $\begin{array}{c}1^{\mathrm{st}} \\
\text { Season } \\
\end{array}$ & $\begin{array}{c}2^{\text {nd }} \\
\text { Season } \\
\end{array}$ & $\begin{array}{c}1^{\text {st }} \\
\text { Season } \\
\end{array}$ & $\begin{array}{c}2^{\text {nd }} \\
\text { Season } \\
\end{array}$ & $\begin{array}{c}1^{\text {st }} \\
\text { Season } \\
\end{array}$ & $\begin{array}{c}2^{\text {nd }} \\
\text { Season }\end{array}$ & $\begin{array}{c}1^{\text {st }} \\
\text { Season } \\
\end{array}$ & $\begin{array}{c}2^{\text {nd }} \\
\text { Season } \\
\end{array}$ & $\begin{array}{c}1^{\text {st }} \\
\text { Season } \\
\end{array}$ & $\begin{array}{c}2^{\text {nd }} \\
\text { Season } \\
\end{array}$ \\
\hline \multicolumn{13}{|l|}{ Irrigation type } \\
\hline Normal & $48.5 \mathrm{~A}$ & $43.4 \mathrm{~A}$ & $13.9 \mathrm{~A}$ & $13.0 \mathrm{~A}$ & $875.8 \mathrm{~A}$ & $832.8 \mathrm{~A}$ & $59.95 \mathrm{~A}$ & $58.69 \mathrm{~A}$ & $8.93 \mathrm{~A}$ & $8.58 \mathrm{~A}$ & $80.47 \mathrm{~A}$ & $79.35 \mathrm{~A}$ \\
\hline Stress & $47.34 \mathrm{~A}$ & $40.9 \mathrm{~B}$ & $12.1 \mathrm{~B}$ & $12.2 \mathrm{~B}$ & $770.6 \mathrm{~B}$ & $662.6 \mathrm{~B}$ & $48.35 \mathrm{~B}$ & $45.96 \mathrm{~B}$ & $7.72 \mathrm{~B}$ & $6.32 \mathrm{~B}$ & $78.36 \mathrm{~B}$ & $76.93 \mathrm{~B}$ \\
\hline \multicolumn{13}{|l|}{ Seed Treatments } \\
\hline Dry seed & $45.45 \mathrm{~B}$ & $38.65 \mathrm{C}$ & $11.75 \mathrm{~B}$ & $10.90 \mathrm{C}$ & $656.5 \mathrm{C}$ & $609.0 \mathrm{C}$ & $40.65 \mathrm{D}$ & $38.79 \mathrm{D}$ & $6.97 \mathrm{D}$ & $5.56 \mathrm{~B}$ & $76.70 \mathrm{C}$ & $74.93 \mathrm{E}$ \\
\hline Water & $46.75 \mathrm{~B}$ & $40.55 \mathrm{BC}$ & $12.10 \mathrm{~B}$ & $12.15 \mathrm{~B}$ & $682.0 \mathrm{C}$ & $657.0 \mathrm{C}$ & $43.20 \mathrm{C}$ & $39.97 \mathrm{D}$ & $7.65 \mathrm{C}$ & $6.36 \mathrm{~B}$ & $78.21 \mathrm{~B}$ & $77.29 \mathrm{D}$ \\
\hline $\mathrm{Na} 2 \mathrm{HPO} 4$ & $49.30 \mathrm{~A}$ & $44.40 \mathrm{~A}$ & $14.05 \mathrm{~A}$ & $13.80 \mathrm{~A}$ & $1045.0 \mathrm{~A}$ & $890.0 \mathrm{~A}$ & $66.82 \mathrm{~A}$ & $64.88 \mathrm{~A}$ & $10.19 \mathrm{~A}$ & $8.67 \mathrm{~A}$ & $81.14 \mathrm{~A}$ & $80.51 \mathrm{~A}$ \\
\hline $\mathrm{MgSO} 4$ & $49.25 \mathrm{~A}$ & $42.75 A B$ & $13.55 \mathrm{~A}$ & $13.30 \mathrm{AB}$ & $873.0 \mathrm{~B}$ & $809.0 \mathrm{~B}$ & $60.60 \mathrm{~B}$ & $60.65 \mathrm{~B}$ & $8.85 \mathrm{~B}$ & $8.71 \mathrm{~A}$ & $80.69 \mathrm{~A}$ & $79.29 \mathrm{~B}$ \\
\hline $\mathrm{KCl}$ & $48.75 \mathrm{~A}$ & $43.10 \mathrm{AB}$ & $13.60 \mathrm{~A}$ & $12.90 \mathrm{AB}$ & $859.5 \mathrm{~B}$ & $773.5 \mathrm{~B}$ & $59.50 \mathrm{~B}$ & $57.36 \mathrm{C}$ & $7.96 \mathrm{C}$ & $7.97 \mathrm{~A}$ & $80.35 \mathrm{~A}$ & $78.68 \mathrm{C}$ \\
\hline
\end{tabular}

Values within the same column followed by the same letters are not significantly different, using Duncan's Multiple Range Test at $5 \%$ level 
Table (1-b). Effect of interaction between irrigation type (normal\& water stress) and seed priming for $24 \mathrm{~h}$ either in water (hydropriming) or in $3 \%$ $\mathrm{Na}_{2} \mathrm{HPO}_{4}, \mathrm{MgSO}_{4}$, or $\mathrm{KCl}$ solution (osmopriming) on growth criteria of okra (var. Sabahia) plants during summer seasons 2012, 2013. RWC $=$ Relative water contents.

\begin{tabular}{|c|c|c|c|c|c|c|c|c|c|c|c|c|c|}
\hline \multirow{2}{*}{\multicolumn{2}{|c|}{$\begin{array}{l}\text { character } \\
\text { Treatments }\end{array}$}} & \multicolumn{2}{|c|}{ Plant height (cm) } & \multicolumn{2}{|c|}{ No. of leaves/ plant } & \multicolumn{2}{|c|}{ Leaves area/ plant $(\mathrm{cm} 2)$} & \multicolumn{2}{|c|}{ Fresh weight/ plant $(\mathrm{g})$} & \multicolumn{2}{|c|}{ Dry weight/ plant (g) } & \multicolumn{2}{|c|}{ R.W.C. } \\
\hline & & $1^{\text {st }}$ Season & $2^{\text {nd }}$ Season & $1^{\text {st }}$ Season & $2^{\text {nd }}$ Season & $1^{\text {st }}$ Season & $2^{\text {nd }}$ Season & $1^{\text {st }}$ Season & $2^{\text {nd }}$ Season & $1^{\text {st }}$ Season & $2^{\text {nd }}$ Season & $1^{\text {st }}$ Season & $2^{\text {nd }}$ Season \\
\hline \multirow{5}{*}{ Normal } & Dry & $46.0 \mathrm{BCD}$ & $40.3 \mathrm{CDE}$ & $12.7 \mathrm{CD}$ & $11.9 \mathrm{~B}$ & $753 \mathrm{D}$ & $720 \mathrm{C}$ & $42.10 \mathrm{E}$ & $39.90 \mathrm{EF}$ & 7.16 DE & $5.86 \mathrm{BCD}$ & $78.60 \mathrm{D}$ & $76.31 \mathrm{~F}$ \\
\hline & Water & $47.8 \mathrm{ABC}$ & $42.1 \mathrm{ABCD}$ & $13.0 \mathrm{BC}$ & $12.5 \mathrm{AB}$ & $765 \mathrm{D}$ & $751 \mathrm{C}$ & $44.90 \mathrm{D}$ & $42.10 \mathrm{E}$ & $7.95 \mathrm{CD}$ & $7.03 \mathrm{BC}$ & $79.68 \mathrm{CD}$ & $78.45 \mathrm{D}$ \\
\hline & $\mathrm{Na}_{2} \mathrm{HPO}_{4}$ & $50.0 \mathrm{~A}$ & $45.9 \mathrm{~A}$ & $15.0 \mathrm{~A}$ & $14.0 \mathrm{~A}$ & $1105 \mathrm{~A}$ & $990 \mathrm{~A}$ & $80.30 \mathrm{~A}$ & $78.50 \mathrm{~A}$ & $11.79 \mathrm{~A}$ & $10.48 \mathrm{~A}$ & $81.87 \mathrm{~A}$ & $81.40 \mathrm{~A}$ \\
\hline & $\mathrm{MgSO} 4$ & $49.5 \mathrm{~A}$ & $44.7 \mathrm{AB}$ & $14.5 \mathrm{AB}$ & $13.7 \mathrm{AB}$ & $886 \mathrm{C}$ & $863 \mathrm{~B}$ & $66.45 \mathrm{~B}$ & $68.75 \mathrm{~B}$ & $8.93 \mathrm{~B}$ & $10.09 \mathrm{~A}$ & $81.27 \mathrm{AB}$ & $80.47 \mathrm{~B}$ \\
\hline & $\mathrm{KCL}$ & $49.0 \mathrm{~A}$ & $44.1 \mathrm{ABC}$ & $14.5 \mathrm{AB}$ & $13.0 \mathrm{AB}$ & $870 \mathrm{C}$ & $840 \mathrm{~B}$ & 66.02 B & $64.22 \mathrm{C}$ & $8.81 \mathrm{~B}$ & $9.43 \mathrm{~A}$ & $80.94 \mathrm{ABC}$ & $80.10 B C$ \\
\hline \multirow{5}{*}{ Stress } & Dry & $44.9 \mathrm{D}$ & $37.0 \mathrm{E}$ & $10.8 \mathrm{E}$ & $9.9 \mathrm{C}$ & $560 \mathrm{E}$ & $498 \mathrm{D}$ & $39.19 \mathrm{~F}$ & $37.69 \mathrm{~F}$ & $6.77 \mathrm{E}$ & $5.25 \mathrm{D}$ & $74.80 \mathrm{~F}$ & $73.55 \mathrm{G}$ \\
\hline & Water & $45.7 \mathrm{CD}$ & $39.0 \mathrm{DE}$ & 11.2 DE & $11.8 \mathrm{~B}$ & $599 \mathrm{E}$ & $563 \mathrm{D}$ & $41.50 \mathrm{E}$ & $37.83 \mathrm{~F}$ & 7.35 DE & $5.69 \mathrm{CD}$ & $76.73 \mathrm{E}$ & $76.14 \mathrm{~F}$ \\
\hline & $\mathrm{Na}_{2} \mathrm{HPO}_{4}$ & $48.6 \mathrm{AB}$ & 42.9 ABCD & $13.1 \mathrm{BC}$ & $13.6 \mathrm{AB}$ & $985 \mathrm{~B}$ & $790 \mathrm{BC}$ & $53.35 \mathrm{C}$ & $51.25 \mathrm{D}$ & $8.59 \mathrm{BC}$ & $6.85 \mathrm{BC}$ & $80.40 \mathrm{BC}$ & $79.62 \mathrm{C}$ \\
\hline & $\mathrm{MgSO} 4$ & $49.0 \mathrm{~A}$ & $43.8 \mathrm{ABC}$ & $12.6 \mathrm{CD}$ & $12.9 \mathrm{AB}$ & $860 \mathrm{C}$ & $755 \mathrm{C}$ & $54.75 \mathrm{C}$ & $52.55 \mathrm{D}$ & $8.77 \mathrm{~B}$ & $7.32 \mathrm{~B}$ & $80.12 B C$ & $78.11 \mathrm{D}$ \\
\hline & $\mathrm{KCL}$ & $48.5 \mathrm{ABC}$ & $42.0 \mathrm{ABCD}$ & $12.7 \mathrm{CD}$ & $12.8 \mathrm{AB}$ & $849 \mathrm{C}$ & $707 \mathrm{C}$ & $52.97 \mathrm{C}$ & $50.50 \mathrm{D}$ & $7.11 \mathrm{E}$ & $6.51 \mathrm{BCD}$ & $79.76 \mathrm{CD}$ & $77.25 \mathrm{E}$ \\
\hline
\end{tabular}

Values within the same column followed by the same letters are not significantly different, using Duncan's Multiple Range Test at $5 \%$ level 
Table (2-a). Effect of irrigation type (normal\& water stress) and seed priming for $24 \mathrm{~h}$ either in water (hydropriming) or in $3 \% \mathrm{Na}_{2} \mathrm{HPO}_{4}$, $\mathrm{MgSO}{ }_{4}$, or $\mathrm{KCl}$ solution (osmopriming) on chlorophyll and total pigments of okra plant leaves (var. Sabahia) during summer seasons 2012, 2013.

\begin{tabular}{|c|c|c|c|c|c|c|c|c|c|c|}
\hline \multirow{2}{*}{$\begin{array}{l}\text { Character } \\
\text { Treatments }\end{array}$} & \multicolumn{2}{|c|}{ Chlorophyll "a" } & \multicolumn{2}{|c|}{ Chlorophyll "b" } & \multicolumn{2}{|c|}{ Chlorophyll "a+b" } & \multicolumn{2}{|c|}{ Carotenoids } & \multicolumn{2}{|c|}{ Total pigments } \\
\hline & $1^{\text {st }}$ Season & $2^{\text {nd }}$ Season & $1^{\text {st }}$ Season & $2^{\text {nd }}$ Season & $1^{\text {st }}$ Season & $2^{\text {nd }}$ Season & $1^{\text {st }}$ Season & $2^{\text {nd }}$ Season & $1^{\text {st }}$ Season & $2^{\text {nd }}$ Season \\
\hline \multicolumn{11}{|l|}{ Varieties } \\
\hline Normal & $10.57 \mathrm{~A}$ & $12.50 \mathrm{~A}$ & $4.36 \mathrm{~B}$ & $4.29 \mathrm{~B}$ & $14.90 \mathrm{~A}$ & $16.82 \mathrm{~A}$ & $1.002 \mathrm{~A}$ & $1.091 \mathrm{~A}$ & $15.94 \mathrm{~A}$ & $17.91 \mathrm{~A}$ \\
\hline Stress & $9.54 \mathrm{~B}$ & $11.00 \mathrm{~B}$ & $4.54 \mathrm{~A}$ & $4.90 \mathrm{~A}$ & $14.08 \mathrm{~B}$ & $15.91 \mathrm{~B}$ & $1.034 \mathrm{~A}$ & $0.861 \mathrm{~B}$ & $15.11 \mathrm{~B}$ & $16.77 \mathrm{~B}$ \\
\hline \multicolumn{11}{|l|}{ Seed Treatments } \\
\hline Dry seed & $8.66 \mathrm{~B}$ & $9.29 \mathrm{C}$ & $3.14 \mathrm{E}$ & $5.44 \mathrm{~B}$ & $11.79 \mathrm{C}$ & $14.73 \mathrm{C}$ & $1.695 \mathrm{~A}$ & $0.775 \mathrm{C}$ & $13.48 \mathrm{C}$ & $15.50 \mathrm{C}$ \\
\hline Water & $9.14 \mathrm{~B}$ & $11.58 \mathrm{~B}$ & $5.39 \mathrm{~A}$ & $5.56 \mathrm{~A}$ & $14.52 \mathrm{~B}$ & $17.14 \mathrm{~A}$ & $0.890 \mathrm{C}$ & $0.790 \mathrm{C}$ & $15.41 \mathrm{~B}$ & $17.93 \mathrm{~A}$ \\
\hline $\mathrm{Na}_{2} \mathrm{HPO}_{4}$ & $11.25 \mathrm{~A}$ & $13.25 \mathrm{~A}$ & $4.59 \mathrm{C}$ & $3.54 \mathrm{E}$ & $15.84 \mathrm{~A}$ & $16.79 \mathrm{~A}$ & $1.190 \mathrm{~B}$ & $1.080 \mathrm{~B}$ & $17.03 \mathrm{~A}$ & $17.87 \mathrm{~A}$ \\
\hline $\mathrm{MgSO} 4$ & $10.79 \mathrm{~A}$ & $13.03 \mathrm{~A}$ & $4.98 \mathrm{~B}$ & $4.36 \mathrm{C}$ & $15.76 \mathrm{~A}$ & $17.39 \mathrm{~A}$ & $0.620 \mathrm{D}$ & $1.215 \mathrm{~A}$ & $16.39 \mathrm{AB}$ & $18.60 \mathrm{~A}$ \\
\hline $\mathrm{KCl}$ & $10.44 \mathrm{~A}$ & $11.70 \mathrm{~B}$ & $4.17 \mathrm{D}$ & $4.09 \mathrm{D}$ & $14.61 \mathrm{~B}$ & $15.79 \mathrm{~B}$ & $0.095 \mathrm{D}$ & $1.025 \mathrm{~B}$ & $15.31 \mathrm{~B}$ & $16.82 \mathrm{~B}$ \\
\hline
\end{tabular}

Values within the same column followed by the same letters are not significantly different, using Duncan's Multiple Range Test at $5 \%$ level 
Table (2-b). Effect of interaction between irrigation type (normal\& water stress) and seed priming for 24h either in water (hydropriming) or in $3 \% \mathrm{Na}_{2} \mathrm{HPO}_{4}, \mathrm{MgSO}_{4}$, or $\mathrm{KCl}$ solution (osmopriming) on chlorophyll and total pigments of okra plant leaves (var. Sabahia) during summer seasons 2012, 2013.

\begin{tabular}{|c|c|c|c|c|c|c|c|c|c|c|c|}
\hline \multirow{2}{*}{\multicolumn{2}{|c|}{$\begin{array}{l}\text { Character } \\
\text { Treatments }\end{array}$}} & \multicolumn{2}{|c|}{ Chlorophyll "a" } & \multicolumn{2}{|c|}{ Chlorophyll "b" } & \multicolumn{2}{|c|}{ Chlorophyll "a+b" } & \multicolumn{2}{|c|}{ Carotenoids } & \multicolumn{2}{|c|}{ Total pigments } \\
\hline & & $1^{\text {st }}$ Season & $2^{\text {nd }}$ Season & $1^{\text {st }}$ Season & $2^{\text {nd }}$ Season & $1^{\text {st }}$ Season & $2^{\text {nd }}$ Season & $1^{\text {st }}$ Season & $2^{\text {nd }}$ Season & $1^{\text {st }}$ Season & $2^{\text {nd }}$ Season \\
\hline \multirow{5}{*}{ Normal } & Dry seed & $8.80 \mathrm{DE}$ & $10.24 \mathrm{~B}$ & $2.95 \mathrm{G}$ & $6.00 \mathrm{~B}$ & $11.75 \mathrm{C}$ & $16.24 \mathrm{~B}$ & $1.81 \mathrm{~A}$ & $0.66 \mathrm{~F}$ & $13.56 \mathrm{C}$ & $16.90 \mathrm{C}$ \\
\hline & Water & $9.73 \mathrm{CDE}$ & $12.96 \mathrm{~A}$ & $6.91 \mathrm{~A}$ & $4.19 \mathrm{E}$ & $16.64 \mathrm{~A}$ & $17.15 \mathrm{~B}$ & $0.74 \mathrm{D}$ & $1.07 \mathrm{BC}$ & $17.38 \mathrm{~A}$ & $18.22 \mathrm{AB}$ \\
\hline & $\mathrm{Na}_{2} \mathrm{HPO}_{4}$ & $11.99 \mathrm{~A}$ & $13.33 \mathrm{~A}$ & $3.30 \mathrm{~F}$ & $3.90 \mathrm{~F}$ & $15.29 \mathrm{~A}$ & $17.23 \mathrm{~B}$ & $1.62 \mathrm{~B}$ & $0.99 \mathrm{CD}$ & $16.91 \mathrm{~A}$ & $18.22 \mathrm{AB}$ \\
\hline & $\mathrm{MgSO} 4$ & $11.45 \mathrm{AB}$ & $13.17 \mathrm{~A}$ & $4.08 \mathrm{D}$ & $3.18 \mathrm{G}$ & $15.53 \mathrm{~A}$ & $16.35 \mathrm{~B}$ & $0.48 \mathrm{E}$ & $1.66 \mathrm{~A}$ & $16.01 \mathrm{AB}$ & $18.01 \mathrm{ABC}$ \\
\hline & $\mathrm{KCl}$ & $10.90 \mathrm{ABC}$ & $12.96 \mathrm{~A}$ & $4.56 \mathrm{C}$ & $4.19 \mathrm{E}$ & $15.46 \mathrm{~A}$ & $17.15 \mathrm{~B}$ & $0.36 \mathrm{~F}$ & $1.07 \mathrm{BC}$ & $15.82 \mathrm{AB}$ & $18.22 \mathrm{AB}$ \\
\hline \multirow{5}{*}{ Stress } & Dry seed & $8.51 \mathrm{E}$ & $8.33 \mathrm{C}$ & $3.32 \mathrm{~F}$ & $4.88 \mathrm{D}$ & $11.83 \mathrm{C}$ & $13.21 \mathrm{D}$ & $1.58 \mathrm{~B}$ & $0.89 \mathrm{D}$ & $13.41 \mathrm{C}$ & $14.10 \mathrm{E}$ \\
\hline & Water & $8.54 \mathrm{E}$ & $10.20 \mathrm{~B}$ & $3.87 \mathrm{DE}$ & $6.93 \mathrm{~A}$ & $12.41 \mathrm{BC}$ & $17.13 \mathrm{~B}$ & $1.04 \mathrm{C}$ & $0.51 \mathrm{G}$ & $13.45 \mathrm{C}$ & $17.64 \mathrm{BC}$ \\
\hline & $\mathrm{Na}_{2} \mathrm{HPO}_{4}$ & $10.51 \mathrm{ABC}$ & $13.17 \mathrm{~A}$ & $5.87 \mathrm{~B}$ & $3.18 \mathrm{G}$ & $16.38 \mathrm{~A}$ & $16.35 \mathrm{~B}$ & $0.76 \mathrm{D}$ & $1.17 \mathrm{~B}$ & $17.14 \mathrm{~A}$ & $17.52 \mathrm{BC}$ \\
\hline & $\mathrm{MgSO} 4$ & $10.13 \mathrm{BCD}$ & $12.90 \mathrm{~A}$ & $5.87 \mathrm{~B}$ & $5.53 \mathrm{C}$ & $16.00 \mathrm{~A}$ & $18.43 \mathrm{~A}$ & $0.76 \mathrm{D}$ & $0.77 \mathrm{E}$ & $16.76 \mathrm{~A}$ & $19.20 \mathrm{~A}$ \\
\hline & $\mathrm{KCl}$ & $9.99 \mathrm{BCDE}$ & $10.44 \mathrm{~B}$ & $3.77 \mathrm{E}$ & $3.99 \mathrm{~F}$ & $13.76 \mathrm{~B}$ & $14.43 \mathrm{C}$ & $1.03 \mathrm{C}$ & $0.98 \mathrm{CD}$ & $14.79 \mathrm{BC}$ & $15.41 \mathrm{D}$ \\
\hline
\end{tabular}

Values within the same column followed by the same letters are not significantly different, using Duncan's Multiple Range Test at $5 \%$ level 
Table (3-a). Effect of irrigation type (normal\& water stress) and seed priming for $24 \mathrm{~h}$ either in water (hydropriming) or in $3 \% \mathrm{Na}_{2} \mathrm{HPO}_{4}$, $\mathrm{MgSO}{ }_{4}$, or $\mathrm{KCl}$ solution (osmopriming) on seed yield of okra (var. Sabahia) during summer seasons 2012, 2013.

\begin{tabular}{|c|c|c|c|c|c|c|c|c|c|c|c|c|}
\hline \multirow[t]{2}{*}{$\begin{array}{l}\text { Character } \\
\text { Treatments }\end{array}$} & \multicolumn{2}{|c|}{ No. of branches/ plant } & \multicolumn{2}{|c|}{ No. of pods/ plant } & \multicolumn{2}{|c|}{$\begin{array}{l}\text { Wt. of seeds/ plant } \\
\text { (g) }\end{array}$} & \multicolumn{2}{|c|}{$\begin{array}{l}\text { Seed yield /plot } \\
(\mathrm{kg})\end{array}$} & \multicolumn{2}{|c|}{ No. of seeds/ pod } & \multicolumn{2}{|c|}{$\begin{array}{l}\text { Wt. of } 100 \text { seeds } \\
\text { (g) }\end{array}$} \\
\hline & $1^{\text {st }}$ Season & $2^{\text {nd }}$ Season & $1^{\text {st }}$ Season & $2^{\text {nd }}$ Season & $1^{\text {st }}$ Season & $2^{\text {nd }}$ Season & $1^{\text {st }}$ Season & $2^{\text {nd }}$ Season & $1^{\text {st }}$ Season & $2^{\text {nd }}$ Season & $1^{\text {st }}$ Season & $2^{\text {nd }}$ Season \\
\hline \multicolumn{13}{|l|}{ Varieties } \\
\hline Normal & $6.89 \mathrm{~A}$ & $6.72 \mathrm{~A}$ & $42.04 \mathrm{~A}$ & $41.11 \mathrm{~A}$ & $159.6 \mathrm{~A}$ & $149.0 \mathrm{~A}$ & $3.69 \mathrm{~A}$ & $3.62 \mathrm{~A}$ & $123.6 \mathrm{~A}$ & $115.4 \mathrm{~A}$ & $5.87 \mathrm{~A}$ & $5.79 \mathrm{~A}$ \\
\hline Stress & $6.31 \mathrm{~B}$ & $5.96 \mathrm{~B}$ & $36.00 \mathrm{~B}$ & $34.00 \mathrm{~B}$ & $121.8 \mathrm{~B}$ & $117.8 \mathrm{~B}$ & $3.47 \mathrm{~B}$ & $3.56 \mathrm{~B}$ & $111.2 \mathrm{~B}$ & $102.2 \mathrm{~B}$ & $5.32 \mathrm{~B}$ & $5.33 \mathrm{~B}$ \\
\hline \multicolumn{13}{|l|}{ Seed Treatments } \\
\hline Dry seed & $6.20 \mathrm{D}$ & $5.98 \mathrm{~B}$ & $34.70 \mathrm{D}$ & $32.60 \mathrm{D}$ & $118.5 \mathrm{E}$ & $110.0 \mathrm{E}$ & $3.37 \mathrm{D}$ & $3.44 \mathrm{C}$ & $99.0 \mathrm{C}$ & $92.5 \mathrm{D}$ & $5.33 \mathrm{~B}$ & $5.31 \mathrm{~B}$ \\
\hline Water & $6.30 \mathrm{D}$ & $6.14 \mathrm{~B}$ & $36.85 \mathrm{C}$ & $36.50 \mathrm{C}$ & $124.9 \mathrm{D}$ & $116.2 \mathrm{D}$ & $3.48 \mathrm{C}$ & $3.54 \mathrm{~B}$ & $106.0 \mathrm{C}$ & $100.0 \mathrm{C}$ & $5.39 \mathrm{~B}$ & $5.37 \mathrm{~B}$ \\
\hline $\mathrm{Na}_{2} \mathrm{HPO}_{4}$ & $7.14 \mathrm{~A}$ & $6.68 \mathrm{~A}$ & $43.55 \mathrm{~A}$ & $42.00 \mathrm{~A}$ & $172.9 \mathrm{~A}$ & $159.6 \mathrm{~A}$ & $3.71 \mathrm{~A}$ & $3.71 \mathrm{~A}$ & $134.0 \mathrm{~A}$ & $123.0 \mathrm{~A}$ & $5.81 \mathrm{~A}$ & $5.73 \mathrm{~A}$ \\
\hline $\mathrm{MgSO} 4$ & $6.80 \mathrm{~B}$ & $6.58 \mathrm{~A}$ & $40.55 \mathrm{~B}$ & $39.2 \mathrm{~B}$ & $150.4 \mathrm{~B}$ & $143.9 \mathrm{~B}$ & $3.70 \mathrm{AB}$ & $3.66 \mathrm{~A}$ & $131.5 \mathrm{~A}$ & $120.0 \mathrm{~A}$ & $5.76 \mathrm{~A}$ & $5.72 \mathrm{~A}$ \\
\hline $\mathrm{KCl}$ & $6.59 \mathrm{C}$ & $6.34 \mathrm{AB}$ & $39.47 \mathrm{~B}$ & $37.45 \mathrm{BC}$ & $136.8 \mathrm{C}$ & $137.4 \mathrm{C}$ & $3.65 \mathrm{~B}$ & $3.63 \mathrm{~A}$ & $116.5 \mathrm{~B}$ & $108.5 \mathrm{~B}$ & $5.71 \mathrm{~A}$ & $5.68 \mathrm{~A}$ \\
\hline
\end{tabular}

Values within the same column followed by the same letters are not significantly different, using Duncan's Multiple Range Test at $5 \%$ level 
Table (3-b). Effect of interaction between irrigation type (normal\& water stress) and seed priming for $24 \mathrm{~h}$ either in water (hydropriming) or in 3\% $\mathrm{Na}_{2} \mathrm{HPO}_{4}, \mathrm{MgSO}_{4}$, or $\mathrm{KCl}$ solution (osmopriming) seed yield of okra (var. Sabahia) during summer seasons 2012, 2013.

\begin{tabular}{|c|c|c|c|c|c|c|c|c|c|c|c|c|c|}
\hline \multirow{2}{*}{\multicolumn{2}{|c|}{$\begin{array}{l}\text { Character } \\
\text { Treatments }\end{array}$}} & \multicolumn{2}{|c|}{ No. of branches/ plant } & \multicolumn{2}{|c|}{ No. of pods/ plant } & \multicolumn{2}{|c|}{$\begin{array}{l}\text { Wt. of seeds/ plant } \\
\text { (g) }\end{array}$} & \multicolumn{2}{|c|}{$\begin{array}{l}\text { Seed yield / plot } \\
\qquad(\mathrm{kg})\end{array}$} & \multicolumn{2}{|c|}{ No. of seeds/ pod } & \multicolumn{2}{|c|}{$\begin{array}{l}\text { Wt. of } 100 \text { seeds } \\
(\mathrm{g})\end{array}$} \\
\hline & & $1^{\text {st }}$ Season & $2^{\text {nd }}$ Season & $1^{\text {st }}$ Season & $2^{\text {nd }}$ Season & $1^{\text {st }}$ Season & $2^{\text {nd }}$ Season & $1^{\text {st }}$ Season & $2^{\text {nd }}$ Season & $1^{\text {st }}$ Season & $2^{\text {nd }}$ Season & $1^{\text {st }}$ Season & $2^{\text {nd }}$ Season \\
\hline \multirow{5}{*}{ Normal } & Dry seed & $6.60 \mathrm{D}$ & $6.42 \mathrm{BCD}$ & 38.5 DE & $36.2 \mathrm{C}$ & 142.7 D & $131.8 \mathrm{D}$ & $3.53 \mathrm{D}$ & $3.45 \mathrm{C}$ & 104.0 DEF & $96.0 \mathrm{C}$ & $5.73 \mathrm{BC}$ & $5.70 \mathrm{BCD}$ \\
\hline & Water & $6.67 \mathrm{CD}$ & $\begin{array}{l}6.51 \\
A B C D\end{array}$ & $40.5 \mathrm{CD}$ & $41.9 \mathrm{AB}$ & 146.1 D & $135.0 \mathrm{D}$ & $3.62 \mathrm{C}$ & $3.59 \mathrm{~B}$ & $112.0 \mathrm{CDE}$ & $105.0 \mathrm{~B}$ & $5.75 \mathrm{BC}$ & $5.73 \mathrm{ABC}$ \\
\hline & $\mathrm{Na}_{2} \mathrm{HPO}_{4}$ & $7.31 \mathrm{~A}$ & $7.05 \mathrm{~A}$ & $45.9 \mathrm{~A}$ & $44.0 \mathrm{~A}$ & $185.9 \mathrm{~A}$ & $173.3 \mathrm{~A}$ & $3.78 \mathrm{AB}$ & $3.72 \mathrm{~A}$ & $143.0 \mathrm{~A}$ & $134.0 \mathrm{~A}$ & $5.98 \mathrm{~A}$ & $5.86 \mathrm{~A}$ \\
\hline & $\mathrm{MgSO} 4$ & $7.00 \mathrm{~B}$ & $6.92 \mathrm{AB}$ & $43.3 \mathrm{~B}$ & $42.6 \mathrm{AB}$ & $167.7 \mathrm{~B}$ & $155.0 \mathrm{~B}$ & $3.80 \mathrm{~A}$ & $3.69 A B$ & $139.0 \mathrm{~A}$ & $130.0 \mathrm{~A}$ & $5.99 \mathrm{~A}$ & $5.85 A B$ \\
\hline & $\mathrm{KCl}$ & $6.85 \mathrm{BC}$ & $6.70 \mathrm{ABC}$ & $42.0 \mathrm{BC}$ & $40.8 \mathrm{~B}$ & $155.6 \mathrm{C}$ & $149.7 \mathrm{C}$ & $3.71 \mathrm{~B}$ & $3.65 A B$ & $120.0 \mathrm{BC}$ & $112.0 \mathrm{~B}$ & $5.89 A B$ & $5.80 A B$ \\
\hline \multirow{5}{*}{ Stress } & Dry seed & $5.79 \mathrm{~F}$ & $5.53 \mathrm{~F}$ & $30.9 \mathrm{G}$ & $29.0 \mathrm{D}$ & $94.3 \mathrm{H}$ & $88.2 \mathrm{G}$ & $3.20 \mathrm{~F}$ & $3.42 \mathrm{C}$ & $94.0 \mathrm{~F}$ & $89.0 \mathrm{C}$ & $4.93 \mathrm{E}$ & $4.89 \mathrm{E}$ \\
\hline & Water & $5.93 \mathrm{~F}$ & $5.76 \mathrm{EF}$ & $33.2 \mathrm{~F}$ & $31.1 \mathrm{D}$ & $103.8 \mathrm{G}$ & $97.3 \mathrm{~F}$ & $3.33 \mathrm{E}$ & $3.49 \mathrm{C}$ & $100.0 \mathrm{EF}$ & $95.0 \mathrm{C}$ & $5.02 \mathrm{E}$ & $5.01 \mathrm{E}$ \\
\hline & $\mathrm{Na}_{2} \mathrm{HPO}_{4}$ & $6.97 \mathrm{~B}$ & 6.30 BCDE & $41.2 \mathrm{BC}$ & $40.0 \mathrm{~B}$ & $160.0 \mathrm{BC}$ & $145.8 \mathrm{C}$ & $3.63 \mathrm{C}$ & $3.67 \mathrm{AB}$ & $125.0 \mathrm{~B}$ & $112.0 \mathrm{~B}$ & $5.64 \mathrm{CD}$ & $5.59 \mathrm{CD}$ \\
\hline & $\mathrm{MgSO} 4$ & $6.60 \mathrm{D}$ & $6.24 \mathrm{CDE}$ & $37.8 \mathrm{E}$ & $35.8 \mathrm{C}$ & $133.2 \mathrm{E}$ & 132.7 D & $3.59 \mathrm{CD}$ & $3.63 \mathrm{AB}$ & $124.0 \mathrm{BC}$ & $110.0 \mathrm{~B}$ & $5.53 \mathrm{D}$ & $5.59 \mathrm{CD}$ \\
\hline & $\mathrm{KCl}$ & $6.33 \mathrm{E}$ & 5.98 DEF & $36.9 \mathrm{E}$ & $34.1 \mathrm{C}$ & $117.9 \mathrm{~F}$ & $125.0 \mathrm{E}$ & $3.58 \mathrm{CD}$ & $3.60 \mathrm{~B}$ & $113.0 \mathrm{BCD}$ & $105.0 \mathrm{~B}$ & $5.50 \mathrm{D}$ & $5.55 \mathrm{D}$ \\
\hline
\end{tabular}

Values within the same column followed by the same letters are not significantly different, using Duncan's Multiple Range Test at $5 \%$ level 
Table (4-a). Effect of irrigation type (normal\& water stress) and seed priming for $24 \mathrm{~h}$ either in water (hydropriming) or in $3 \% \mathrm{Na}_{2} \mathrm{HPO}_{4}$, $\mathrm{MgSO}{ }_{4}$ or $\mathrm{KCl}$ solution (osmopriming) on N, P, K and proline of okra seeds (var. Sabahia) during summer seasons 2012, 2013.

\begin{tabular}{|c|c|c|c|c|c|c|c|c|}
\hline \multirow{2}{*}{$\begin{array}{l}\text { Character } \\
\text { Treatments }\end{array}$} & \multicolumn{2}{|c|}{ N\% } & \multicolumn{2}{|c|}{$\mathrm{P} \%$} & \multicolumn{2}{|c|}{$\mathrm{K} \%$} & \multicolumn{2}{|c|}{ Proline (mg/1g F.wt) } \\
\hline & $1^{\text {st }}$ Season & $2^{\text {nd }}$ Season & $1^{\text {st }}$ Season & $2^{\text {nd }}$ Season & $1^{\text {st }}$ Season & $2^{\text {nd }}$ Season & $1^{\text {st }}$ Season & $2^{\text {nd }}$ Season \\
\hline \multicolumn{9}{|l|}{ Varieties } \\
\hline Normal & $4.36 \mathrm{~A}$ & $4.39 \mathrm{~A}$ & $1.08 \mathrm{~A}$ & $1.09 \mathrm{~A}$ & $0.80 \mathrm{~A}$ & $0.77 \mathrm{~A}$ & $1.17 \mathrm{~B}$ & $0.85 \mathrm{~B}$ \\
\hline Stress & $4.14 \mathrm{~B}$ & $4.35 \mathrm{~B}$ & $1.01 \mathrm{~B}$ & $1.04 \mathrm{~B}$ & $0.71 \mathrm{~B}$ & $0.66 \mathrm{~B}$ & $2.10 \mathrm{~A}$ & $1.70 \mathrm{~A}$ \\
\hline \multicolumn{9}{|l|}{ Seed Treatments } \\
\hline Dry seed & $3.81 \mathrm{C}$ & $4.20 \mathrm{~B}$ & $1.0 \mathrm{~B}$ & $1.02 \mathrm{~B}$ & $0.69 \mathrm{C}$ & $0.66 \mathrm{~B}$ & $1.07 \mathrm{E}$ & $0.74 \mathrm{E}$ \\
\hline Water & $4.25 \mathrm{~B}$ & $4.22 \mathrm{~B}$ & $1.0 \mathrm{~B}$ & $1.02 \mathrm{~B}$ & $0.74 \mathrm{~B}$ & $0.67 \mathrm{~B}$ & $1.09 \mathrm{D}$ & $0.77 \mathrm{D}$ \\
\hline $\mathrm{Na}_{2} \mathrm{HPO}_{4}$ & $4.55 \mathrm{~A}$ & $4.49 \mathrm{~A}$ & $1.09 \mathrm{~A}$ & $1.09 \mathrm{~A}$ & $0.81 \mathrm{~A}$ & $0.77 \mathrm{~A}$ & $2.13 \mathrm{~A}$ & $1.93 \mathrm{~A}$ \\
\hline $\mathrm{MgSO} 4$ & $4.38 \mathrm{AB}$ & $4.47 \mathrm{~A}$ & $1.08 \mathrm{~A}$ & $1.11 \mathrm{~A}$ & $0.80 \mathrm{~A}$ & $0.76 \mathrm{~A}$ & $2.10 \mathrm{~B}$ & $1.71 \mathrm{~B}$ \\
\hline $\mathrm{KCl}$ & $4.30 \mathrm{~B}$ & $4.47 \mathrm{~A}$ & $1.07 \mathrm{~A}$ & $1.09 \mathrm{~A}$ & $0.77 \mathrm{AB}$ & $0.73 \mathrm{~A}$ & $1.79 \mathrm{C}$ & $1.24 \mathrm{C}$ \\
\hline
\end{tabular}

Values within the same column followed by the same letters are not significantly different, using Duncan's Multiple Range Test at $5 \%$ level 
Table (4-b). Effect of interaction between irrigation type (normal\& water stress) and seed priming for $24 \mathrm{~h}$ either in water (hydropriming) or in $3 \%$ $\mathrm{Na}_{2} \mathrm{HPO}_{4}, \mathrm{MgSO}_{4}$, or KCl solution (osmopriming) on N, P, K and proline of okra seeds (var. Sabahia) during summer seasons 2012, 2013.

\begin{tabular}{|c|c|c|c|c|c|c|c|c|c|}
\hline \multirow{2}{*}{\multicolumn{2}{|c|}{$\begin{array}{l}\text { Character } \\
\text { Treatments }\end{array}$}} & \multicolumn{2}{|c|}{ N\% } & \multicolumn{2}{|c|}{$\mathrm{P} \%$} & \multicolumn{2}{|c|}{$\mathrm{K} \%$} & \multicolumn{2}{|c|}{ Proline (mg/1g F.wt) } \\
\hline & & \multirow{2}{*}{$\frac{1^{\text {st }} \text { Season }}{4.22 \mathrm{~B}}$} & \multirow{2}{*}{$\frac{2^{\text {nd }} \text { Season }}{4.20 \mathrm{C}}$} & \multirow{2}{*}{$\begin{array}{l}1^{\text {st }} \text { Season } \\
1.03 \mathrm{BC}\end{array}$} & \multirow{2}{*}{$\frac{2^{\text {nd }} \text { Season }}{1.05 A B}$} & \multirow{2}{*}{$\frac{1^{\text {st }} \text { Season }}{0.71 \mathrm{DEF}}$} & \multirow{2}{*}{$\frac{2^{\text {nd }} \text { Season }}{0.70 \mathrm{~B}}$} & \multirow{2}{*}{$\frac{1^{\text {st }} \text { Season }}{0.54 \mathrm{I}}$} & \multirow{2}{*}{$\frac{2^{\text {nd }} \text { Season }}{0.39 \mathrm{~J}}$} \\
\hline \multirow{5}{*}{ Normal } & Dry seed & & & & & & & & \\
\hline & Water & $4.24 \mathrm{~B}$ & $4.23 \mathrm{C}$ & $1.02 \mathrm{BC}$ & $1.05 \mathrm{AB}$ & $0.79 \mathrm{BC}$ & $0.71 \mathrm{~B}$ & $0.44 \mathrm{~J}$ & $0.40 \mathrm{I}$ \\
\hline & $\mathrm{Na}_{2} \mathrm{HPO}_{4}$ & $4.66 \mathrm{~A}$ & $4.53 \mathrm{~A}$ & $1.13 \mathrm{~A}$ & $1.11 \mathrm{~A}$ & $0.86 \mathrm{~A}$ & $0.82 \mathrm{~A}$ & $1.72 \mathrm{E}$ & $1.37 \mathrm{D}$ \\
\hline & MgSO4 & $4.35 \mathrm{~B}$ & $4.49 \mathrm{AB}$ & $1.13 \mathrm{~A}$ & $1.13 \mathrm{~A}$ & $0.85 \mathrm{~A}$ & $0.82 \mathrm{~A}$ & $1.66 \mathrm{~F}$ & $1.35 \mathrm{E}$ \\
\hline & $\mathrm{KCl}$ & $4.35 \mathrm{~B}$ & $4.50 \mathrm{AB}$ & $1.10 \mathrm{~A}$ & $1.09 \mathrm{~A}$ & $0.81 \mathrm{AB}$ & $0.80 \mathrm{~A}$ & $1.50 \mathrm{H}$ & $0.75 \mathrm{H}$ \\
\hline \multirow{5}{*}{ Stress } & Dry seed & $3.39 \mathrm{C}$ & $4.19 \mathrm{C}$ & $0.97 \mathrm{C}$ & 0.99 B & $0.66 \mathrm{~F}$ & $0.62 \mathrm{C}$ & $1.60 \mathrm{G}$ & $1.09 \mathrm{G}$ \\
\hline & Water & $4.25 \mathrm{~B}$ & $4.21 \mathrm{C}$ & $0.98 \mathrm{BC}$ & $0.99 \mathrm{~B}$ & $0.68 \mathrm{EF}$ & $0.63 \mathrm{C}$ & $1.73 \mathrm{D}$ & $1.14 \mathrm{~F}$ \\
\hline & $\mathrm{Na}_{2} \mathrm{HPO}_{4}$ & $4.43 \mathrm{AB}$ & $4.45 \mathrm{AB}$ & $1.04 \mathrm{~B}$ & $1.06 \mathrm{AB}$ & $0.75 \mathrm{CD}$ & $0.71 \mathrm{~B}$ & $2.53 \mathrm{~B}$ & $2.49 \mathrm{~A}$ \\
\hline & MgSO4 & $4.40 \mathrm{~B}$ & $4.44 \mathrm{~B}$ & $1.03 \mathrm{BC}$ & $1.09 \mathrm{~A}$ & $0.75 \mathrm{CD}$ & $0.69 \mathrm{~B}$ & $2.54 \mathrm{~A}$ & $2.04 \mathrm{~B}$ \\
\hline & $\mathrm{KCl}$ & $4.25 B$ & $4.44 \mathrm{~B}$ & $1.03 \mathrm{BC}$ & $1.08 \mathrm{~A}$ & $0.72 \mathrm{DE}$ & $0.65 \mathrm{BC}$ & $2.07 \mathrm{C}$ & $1.72 \mathrm{C}$ \\
\hline
\end{tabular}

Values within the same column followed by the same letters are not significantly different, using Duncan's Multiple Range Test at $5 \%$ level 
Table (5-a). Effect of irrigation type (normal\& water stress) and seed priming for $24 \mathrm{~h}$ either in water (hydropriming) or in $3 \% \mathrm{Na}_{2} \mathrm{HPO}_{4}$, $\mathrm{MgSO}{ }_{4}$, or $\mathrm{KCl}$ solution (osmopriming) seed quality of okra (var. Sabahia) during summer seasons 2012, 2013.

\begin{tabular}{|c|c|c|c|c|c|c|c|c|}
\hline \multirow[t]{2}{*}{$\begin{array}{l}\text { Character } \\
\text { Treatments }\end{array}$} & \multicolumn{2}{|c|}{ Germination percentage (\%) } & \multicolumn{2}{|c|}{ Rat of germination (day) } & \multicolumn{2}{|c|}{$\begin{array}{l}\text { Seedling length } \\
(\mathrm{cm})\end{array}$} & \multicolumn{2}{|c|}{ Seedling root length $(\mathrm{cm})$} \\
\hline & $1^{\text {st }}$ Season & $2^{\text {nd }}$ Season & $1^{\text {st }}$ Season & $2^{\text {nd }}$ Season & $1^{\text {st }}$ Season & $2^{\text {nd }}$ Season & $1^{\text {st }}$ Season & $2^{\text {nd }}$ Season \\
\hline \multicolumn{9}{|l|}{ Varieties } \\
\hline Normal & $92.03 \mathrm{~A}$ & $93.2 \mathrm{~A}$ & $2.52 \mathrm{~B}$ & $2.54 \mathrm{~B}$ & $26.5 \mathrm{~A}$ & $26.7 \mathrm{~A}$ & $12.5 \mathrm{~A}$ & $12.6 \mathrm{~A}$ \\
\hline Stress & $89.5 \mathrm{~B}$ & $89.0 \mathrm{~B}$ & $2.85 \mathrm{~A}$ & $2.79 \mathrm{~A}$ & $22.0 \mathrm{~B}$ & $20.9 \mathrm{~B}$ & $10.46 \mathrm{~B}$ & $10.3 \mathrm{~B}$ \\
\hline \multicolumn{9}{|l|}{ Seed Treatments } \\
\hline Dry seed & $89.08 \mathrm{D}$ & $90.25 \mathrm{~B}$ & $2.77 \mathrm{~A}$ & $2.75 \mathrm{~A}$ & $22.50 \mathrm{~B}$ & $22.25 \mathrm{C}$ & $9.50 \mathrm{~B}$ & $9.25 \mathrm{D}$ \\
\hline Water & $90.00 \mathrm{CD}$ & $90.50 \mathrm{AB}$ & $2.76 \mathrm{~A}$ & $2.75 \mathrm{~A}$ & $23.75 \mathrm{AB}$ & $22.75 \mathrm{BC}$ & $10.0 \mathrm{~B}$ & $10.5 \mathrm{C}$ \\
\hline $\mathrm{Na}_{2} \mathrm{HPO}_{4}$ & $92.25 \mathrm{~A}$ & $92.00 \mathrm{~A}$ & $2.58 \mathrm{C}$ & $2.60 \mathrm{~B}$ & $25.50 \mathrm{~A}$ & $25.25 \mathrm{~A}$ & $13.25 \mathrm{~A}$ & $13.25 \mathrm{~A}$ \\
\hline MgSO4 & $91.50 \mathrm{AB}$ & $91.75 \mathrm{AB}$ & $2.65 \mathrm{~B}$ & $2.60 \mathrm{~B}$ & $25.00 \mathrm{~A}$ & $24.50 \mathrm{AB}$ & $12.50 \mathrm{~A}$ & $12.00 \mathrm{~B}$ \\
\hline $\mathrm{KCL}$ & $91.00 \mathrm{BC}$ & $91.00 \mathrm{AB}$ & $2.68 \mathrm{~B}$ & $2.63 \mathrm{~B}$ & $24.50 \mathrm{AB}$ & $24.25 \mathrm{ABC}$ & $12.15 \mathrm{~A}$ & $12.25 \mathrm{~B}$ \\
\hline
\end{tabular}

Values within the same column followed by the same letters are not significantly different, using Duncan's Multiple Range Test at $5 \%$ level 
Table (5-b). Effect of interaction between irrigation type (normal\& water stress) and seed priming for $24 \mathrm{~h}$ either in water (hydropriming) or in $3 \%$ $\mathrm{Na}_{2} \mathrm{HPO}_{4}, \mathrm{MgSO}_{4}$, or $\mathrm{KCl}$ solution (osmopriming) seed quality of okra (var. Sabahia) during summer seasons 2012, 2013.

\begin{tabular}{|c|c|c|c|c|c|c|c|c|c|}
\hline \multicolumn{2}{|c|}{$\begin{array}{l}\text { Character } \\
\text { Treatments }\end{array}$} & \multicolumn{2}{|c|}{ Germination percentage (\%) } & \multicolumn{2}{|c|}{ Rate of germination (day) } & \multicolumn{2}{|c|}{$\begin{array}{l}\text { Seedling length } \\
(\mathrm{cm})\end{array}$} & \multicolumn{2}{|c|}{ Seedling root length $(\mathrm{cm})$} \\
\hline & & $1^{\text {st }}$ Season & $2^{\text {nd }}$ Season & $1^{\text {st }}$ Season & $2^{\text {nd }}$ Season & $1^{\text {st }}$ Season & $2^{\text {nd }}$ Season & $1^{\text {st }}$ Season & $2^{\text {nd }}$ Season \\
\hline \multirow{5}{*}{ Normal } & Dry seed & $91.17 \mathrm{BC}$ & $92.00 \mathrm{BC}$ & $2.58 \mathrm{D}$ & $2.65 \mathrm{C}$ & $25.0 \mathrm{ABC}$ & $25.0 \mathrm{~B}$ & $11.0 \mathrm{CD}$ & $10.0 \mathrm{DE}$ \\
\hline & Water & $91.50 \mathrm{BC}$ & $92.50 \mathrm{AB}$ & $2.60 \mathrm{D}$ & $2.60 \mathrm{CD}$ & $26.0 \mathrm{AB}$ & $25.0 \mathrm{~B}$ & $11.0 \mathrm{CD}$ & $11.5 \mathrm{C}$ \\
\hline & $\mathrm{Na}_{2} \mathrm{HPO}_{4}$ & $93.50 \mathrm{~A}$ & $94.00 \mathrm{AB}$ & $2.45 \mathrm{E}$ & $2.50 \mathrm{DE}$ & $27.5 \mathrm{~A}$ & $28.5 \mathrm{~A}$ & $14.0 \mathrm{~A}$ & $15.0 \mathrm{~A}$ \\
\hline & $\mathrm{MgSO} 4$ & $92.00 \mathrm{AB}$ & $94.50 \mathrm{~A}$ & $2.48 \mathrm{E}$ & $2.50 \mathrm{DE}$ & $27.0 \mathrm{~A}$ & $27.5 \mathrm{AB}$ & $13.5 \mathrm{AB}$ & $13.0 \mathrm{~B}$ \\
\hline & $\mathrm{KCL}$ & $92.00 \mathrm{AB}$ & $93.00 \mathrm{AB}$ & $2.50 \mathrm{E}$ & $2.45 \mathrm{E}$ & $27.0 \mathrm{~A}$ & $27.50 \mathrm{AB}$ & $13.0 \mathrm{ABC}$ & $13.5 \mathrm{~B}$ \\
\hline \multirow{5}{*}{ Stress } & Dry seed & $87.00 \mathrm{E}$ & $88.50 \mathrm{D}$ & $2.95 \mathrm{~A}$ & $2.85 \mathrm{~A}$ & $20.0 \mathrm{E}$ & $19.5 \mathrm{C}$ & $8.0 \mathrm{E}$ & $8.5 \mathrm{~F}$ \\
\hline & Water & 88.50 DE & $88.50 \mathrm{D}$ & $2.91 \mathrm{~A}$ & $2.90 \mathrm{~A}$ & $21.5 \mathrm{DE}$ & $20.5 \mathrm{C}$ & $9.0 \mathrm{DE}$ & $9.5 \mathrm{EF}$ \\
\hline & $\mathrm{Na}_{2} \mathrm{HPO}_{4}$ & $91.00 \mathrm{BC}$ & $90.00 \mathrm{CD}$ & $2.70 \mathrm{C}$ & $2.70 \mathrm{BC}$ & $23.5 \mathrm{BCD}$ & $22.0 \mathrm{C}$ & $12.5 \mathrm{ABC}$ & $11.5 \mathrm{C}$ \\
\hline & $\mathrm{MgSO} 4$ & $91.00 \mathrm{BC}$ & $89.00 \mathrm{D}$ & $2.82 \mathrm{~B}$ & $2.70 \mathrm{BC}$ & $23.0 \mathrm{BCD}$ & $21.5 \mathrm{C}$ & $11.5 \mathrm{BC}$ & $11.0 \mathrm{CD}$ \\
\hline & $\mathrm{KCL}$ & $90.00 \mathrm{CD}$ & $89.00 \mathrm{D}$ & $2.85 \mathrm{~B}$ & $2.80 \mathrm{AB}$ & $22.0 \mathrm{CDE}$ & $21.0 \mathrm{C}$ & $11.3 \mathrm{C}$ & $11.0 \mathrm{CD}$ \\
\hline
\end{tabular}

Values within the same column followed by the same letters are not significantly different, using Duncan's Multiple Range Test at $5 \%$ level 


\section{REFERENCES}

1. A.O.A.C. 2000. Official Methods of Analysis of the Association of Official Agricultural Chemists. $17^{\text {th }}$ Ed, Washington. D.C., USA.

2. Adebisi MA, Kehinde TO, Abdul-Rafiu M A, Esuruoso OA, Oni OD, Ativie O. 2013. Seed physiological quality of three Capsicum species as affected by seed density and hydropriming treatment durations. J. Agron. 12(1): 38-45.

3. Adelakun OE, Oyelade OJ. 2011. Chapter 19 - Potential use of okra seed (Abelmoschus esculentus Moench) flour for food fortification and effects of processing. Flour and breads and their fortification in health and disease prevention. 205-212.

4. Anjum SA, Farooq M, Xie XY, Liu XJ, Ijaz MF. 2012. Antioxidant defense system and proline accumulation enables hot pepper to perform better under drought. Sci. Hortic. 140:66-73.

5. Badek B, Duijn B van, Grzesik M. 2006. Effects of water supply methods and seed moisture content on germination of China aster (Callistephus chinensis) and tomato (Lycopersicon esculentun Mill.) seeds. Eur. J. Agron. 24 (1): 45-51.

6. Barrs HD, Weatherley PE. 1962. A re-examination of the relative turgidity technique for estimating water deficits in leaves. Aust. J Biol. Sci. 15: 413-428.

7. Bates L, waldrenn R, Teare I. 1973. Rapid determination of free proline for water-stress studies. Plant Soil. 39: 205-207.

8. Chen K, Arora R, Arora U. 2010. Osmopriming of spinach (Spinacia oleracea L. $\mathrm{cv}$. Bloomsdale) seeds and germination performance under temperature and water stress. Seed Science and Tech. 38 (1): 36-48.

9. Chen K, Arora R. 2013. Priming memory invokes seed stress-tolerance Original Research Article. Environ. Exper. Botany 94: 33-45.

10. Chen K. Arora R. 2011. Dynamics of the antioxidant system during seed osmopriming, post-priming germination, and seedling establishment in Spinach (Spinacia oleracea). Plant Sci. 180 (2): 212-220.

11. Dospekhov PA. 1984. Field Experimentations and Statistical Procedures, Mir Publishers Moscow.

12. Farhoudi R, Saeedipour S, Mohammadreza D. . 2011. The effect of $\mathrm{NaCl}$ seed priming on salt tolerance, antioxidant enzyme activity, proline and carbohydrate accumulation of Muskmelon (Cucumis melo L.) under saline condition. African J. Agric. Res6(6): 1363-1370. 
13. Farooq M, Basra SMA, Abdul Wahid, Ahmad N. 2010. Changes in nutrienthomeostasis and reserves metabolism during rice seed priming: Consequences for seedling emergence and growth. Agric. Sci. in China 9 (2): 191-198.

14. Ivanice F. dos Santos; Ana M.P. dos Santos; Uenderson A. Barbosa; Jeane S. Lima; Débora C. dos Santos and Geraldo D. Matos (2013). Multivariate analysis of the mineral content of raw and cooked okra (Abelmoschus esculentus L.). Microchem. J. 110: 439-443.

15. Lima LB de, Marcos FJ. 2010. Cucumber (Cucumis sativus) seed priming methods and germination at different temperatures. [Portuguese]. Revista Brasileira de Sementes. 32(1):138-147.

16. Rahimi A. 2013. Seed priming improves the germination performance of cumin (Cuminum syminum L.) under temperature and water stress. Indust. Crops and Products. 42: 454-460.

17. Rajavel M, Vincent S. 2009. Effect of seed hardening of pulses for drought tolerance. J. Ecobiol. 24(3): 293-299.

18. Rastin S, Madani H, Shoaei S. 2013. Effect of seed priming on red bean (Phaseolus calcaratus) growth and yield. Ann. Biol. Res. 4(2): 292-296.

19. Ratikanta KM. 2011. Seed priming: an efficient farmers' technology to improve seedling vigour, seedling establishment and crop productivity. Int. J. Bioresource Stress Manag. 2(3): i-iv.

20. Rehmani HU, Basra SMA, Farooq M. 2011. Field appraisal of seed priming to improve the growth, yield, and quality of direct seeded rice. Turk J Agric. 35: 357-365.

21. Shah AR, Sajid M, Abdur-Rab Ara N, Ahmad M, Wahid F, Shafi G. 2011. Response of germination, growth and yield of okra (Abelmoschus esculentus) to seed priming duration and p-sources in Northwest Pakistan African J. Plant Sci. 5(11): 663-670.

22. Sikhondze DK, Ossom EM. 2011. Impact of priming okra (Abelmoschus esculentus L.) seeds on seedling performance in Swaziland. Adv. Environ. Biol. 5(6):1221-1228.

23. Sivritepe HO, Sivritepe N, Eris A, Turhan E. 2005. The effects of $\mathrm{NaCl}$ pretreatments on salt tolerance of melons grown under long-term salinity. Scientia Hort. 106(4): 568-581.

24. Snedecor GW, Cochran WG. 1980. Statistical Methods. 7th Ed. Lowa State Univ. Press, Amer, Lowa, USA.

25. Umair A, Ali S, Bashir K, Hussain S. 2010. Evaluation of different seed priming techniques in mung bean (Vigna radiata). Soil and Environment. 29(2): 181-186. 


\title{
تأثير معاملات تهيئة البذور للإنبات على النمو \\ و محصول البذور فى الباميا تحت الإجهاد المائي
}

\author{
أمل زكريا حجازي
}

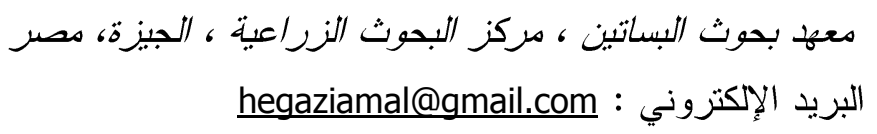

تهيئة البذور هى تقنية تلاحظ أنها تحسن أداء البذور فى الحقل و بالتالي تحسن الإنبات و النمو و محصول البذور • وعلاوة على ذلك ، فإن تهيئة البذور غالبا ما يصاحبها تحسين مقاومة الإجهاد للبذور النابتة. وقد أجريت هذه الدراسة لتحديد محصول البذور و جودة فى صنف محلي من من الباميا

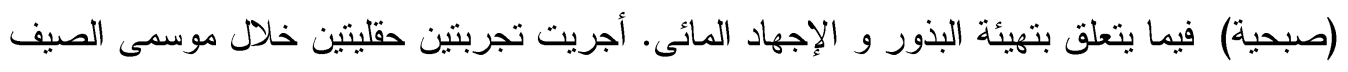

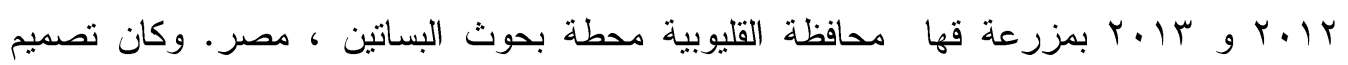
التجربة في قطع منشقة فى أربع مكررات. تمنل القطع الرئيسية نظامين الري إما طبيعية أو الإجهاد

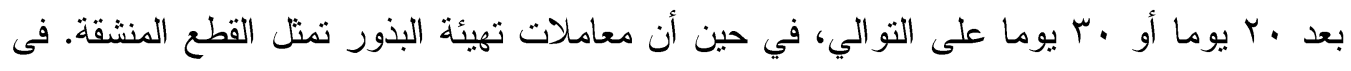

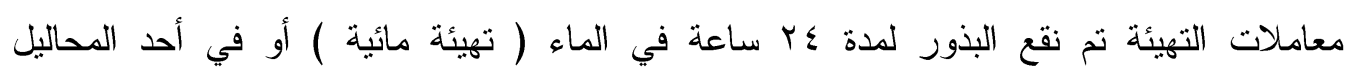
MgSO INa $_{2} \mathrm{HPO}_{4}$ الكنترول. و أنشارت النتائج أن كل الصفات النباتية قد انخفضت تحت الإجهاد المائى في حين تم تحسين هذا

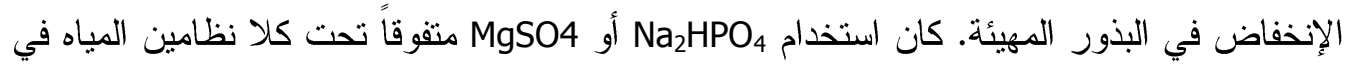
معايير النمو ومحصول البذور و جودنة. وقد أعطت التهيئة ب KCl تحسين إيجابى أقل ولكن كان

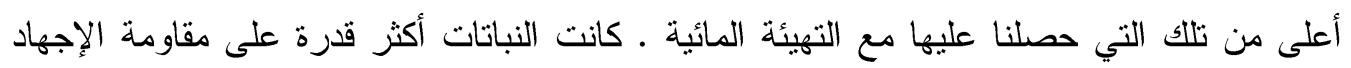
المائى نتيجة لتهيئة البذور التي أدت إلى نراكم بعض osmolytes مثل البرولين ـ أظهرت النتائج

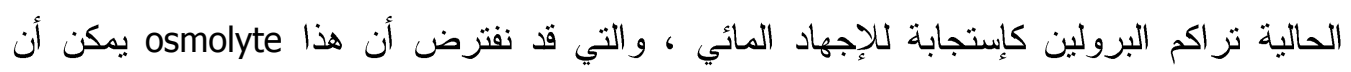

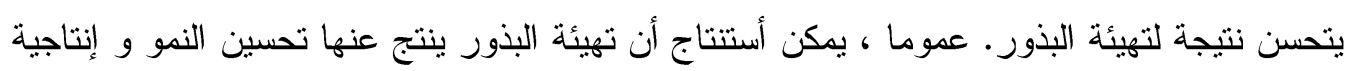

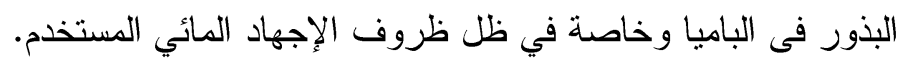

